\title{
A Design of Distributed Mobility Management Architecture in Context-Aware RF Sensor Information Transmission System
}

\author{
Ronnie D. Caytiles and Byungjoo Park* \\ Department of Multimedia Engineering, Hannam University \\ 133 Ojeong-dong, Daeduk-gu, Daejeon, Korea \\ rdcaytiles@gmail.com, „bjpark@hnu.kr
}

\begin{abstract}
Recently, the implementation of wireless structural health monitoring for socialoverhead capital (SOC) public infrastructures has been increasingly become a popular in order to enhance its efficiency and safety. This paper deals with the development of a seamless wireless sensor information transmission system based on Software Defined Networking (SDN) as its distributed mobility management (DMM) support. The advantages of the separation of data and control infrastructures of SDN were utilized in order to optimize the delivery and distribution of critical structural information which will be crucial on the safety of commuters on public bridges as part of SOC public infrastructure. The system is designed in order to enhance the maintenance of the nextgeneration SOC infrastructure that will be capable of realizing and adapting with the current situation utilizing data abstracted from energy-saving ICT genetic biometric wireless sensor technology. A filtering algorithm for in separating critical structural information from insignificant sensor signals was also introduced.
\end{abstract}

Keywords: SOC public structures, structural health monitoring (SHM), filtering algorithm, distributed mobility management (DMM), software defined networking (SDN), smart sensors

\section{Introduction}

The increasing occurrences of tragic incidents in SOC public civil structures like bridges, railroads, national highways, dams, tunnels, buildings, and others caused by natural calamities (e.g., hurricanes, typhoons, earthquakes, global warming, etc...) is becoming an important social issue. The safety and efficiency of such civil structures is essentially important specifically if human's life is at stake. Such tragic incidents have caused numerous casualties and monetary losses from the past years, thus, an effective management system to address the structural health monitoring (SHM) of these civil structures is essentially needed.

In order to provide a continuous structural health monitoring for SOC public civil structures, a permanent array of sensors can be installed which has become a popular solution and approach specifically for bridge structure management. The geographically distributed sensors installed on bridges are capable of monitoring unusual occurrences that could lead in such tragic incidents which ranges from a simple traffic incursion up to major collapse of bridges. However, this approach has offered major drawbacks in terms data acquisitions and data traffic management which were very essential in order to deliver an efficient real-time monitoring of its structural health. The transport of sensed information is a critical factor in

Received (May 21, 2018), Review Result (August 10, 2018), Accepted (August 16, 2018)

* Corresponding Author 
delivering an efficient SHM. Moreover, the approach can be too costly due to a geographically installation of wired sensing devices.

To compensate the drawbacks from this approach, an innovative solution of installing wireless SHM have emerged. It becomes a promising technique that could permanently address the growing social issues of managing SOC public civil structures. Since the monitoring is real-time, sensing devices are required to constantly transmit structural information into the monitoring location for analysis and management. Thus, various installation approaches were developed to address the increasing amount of data and to manage the data traffic during communication.

The peer-to peer communications for the transmission of the collected structural data and weather conditions can be a deterrent to the optimization of delivering an efficient and real-time traffic and weather updates specifically when a human's life is at stake [1]. Moreover, the single point of failure comes as a major disadvantage whenever a centralized control for the management of SHM [2]. The flow of data traffic (collected structural data, occurrence of vehicular accidents, weather conditions, etc.,) in a centralized management can become too heavy or concentrated on the central controller causing the delivery of critical information insignificant.

The centralized management for wireless devices of an SHM can be a sensible approach to address the increasing volume of structural data traffic but it poses a significant number of limitations such as low scalability, reliability issues (i.e., single point of failure), sub-optimal routing, higher packet loss rate, signaling overhead, lack of granularity on the mobility management service, and leads to a more complex network deployment $[2,3]$. In this regard, alternative management solutions and innovations are required in order to address these issues and provide balanced traffic flow specifically on real-time monitoring of the structural health of SOC public structures.

The emergence of distributed management approaches for SHM can be the solution for such inabilities wherein the control and management of individual wireless sensing nodes will be distributed among different SHM subsystems [5]. This paper deals with the deployment of Software Defined Networking (SDN) on the distributed mobility management support for wireless sensor information transmission system. The approach has taken advantage on the programmable features of SDN to integrate on the existing network-based DMM solutions of proxy mobile Internet protocol version 6 (PMIPv6) [6]. SDN provides a separate data infrastructures to optimize the routing of structural health data traffic by distributing the load through mobile anchors or network entities while the control infrastructures were kept centralized. A single logical switch or SDN controller manages the distribution of structural health data traffic.

The rest of this paper is organized as follows: Section 2 provides an overview of the vulnerabilities of social-overhead capital (SOC) public civil infrastructures being exposed to natural calamities; the analysis of several wireless SHM solutions to minimize the failures on SOC public infrastructures were discussed in Section 3; the distributed mobility management in wireless sensor information transmission is presented in Section 4; and the concluding remarks in Section 5.

\section{SOC Bridge Failures and Issues}

Bridges as part of the SOC public structures are critical in the success of any country's economy. Bridge failures and collapses can lead into damages of properties, injuries, and even casualties. That is why, bridge constructions must be taken into a very serious consideration. Moreover, to further the safety and efficiency of bridges, a robust structural health monitoring must be considered. 
Bridge failures can be caused by a number of different factors such as age and deterioration, strong winds, floods, accidents, constructions incidents, design flaws and manufacturing errors, fires, earthquakes, structural issues, etc. Some problems that bridges also have were the management issues that include funding shortages, delays in constructing new bridges and repairs of old ones, and bridges can also become chokepoints or bottlenecks in freeways.

Some of the incidents of bridge failures were identified which have lead into a series of improvements and deployment of efficient structural health monitoring systems. In 1940, the Tacoma Narrows Bridge in the state of Washington in the US dramatically collapsed into Puget Sound caused design errors of aerodynamic instability [7]. An 8-lane, steel truss arch bridge I-35W Mississippi River Bridge in Minneapolis, Minnesota, US had a catastrophic failure on 2007 that have injured more than a hundred and killed 13 people [8]. The catastrophic failure was caused by a design flaw wherein an under designed gusset plate was ripped along a line of rivets accompanied by the weight (i.e., it was rush hour) on the bridge on that time. In 1987, 10 people were killed when the Schoharie Creek Bridge in the state of New York in the US collapsed due to bridge scour at the foundations caused by a heavy rainfall [9]. The incident has served as a motivation for bridge design improvements and stricter bridge inspection procedures.

In Seoul South Korea, 32 people have died and injured 17 others when the Seongsu Bridge over the Han River that links the Seongdong and Gangnam districts had collapsed on October of 1994 [10]. It was reported that it had collapsed in the morning of October 21 when one of its concrete slabs fell caused by the failure of the suspension structure. An improper welding on the steel trusses of the suspension structure under the concrete slab road was seems to be the cause of the structural failure. It was completely redesigned and rebuilt on August of 1997.

Most of the bridges failures and collapses were caused by design and construction errors, age and deterioration, and triggering events such as vehicular accidents, scour, earthquakes, etc. In this regard, initiatives on bridge construction redesigns were made as well as opening of stronger inspection programs. Moreover, monitoring programs have been opened to further the safety and efficiency of bridge users and commuters.

\section{Wireless Structural Health Monitoring Models}

\subsection{Peer-to-peer Mobility Management}

The management of the mobility of sensors and mobile devices in the SHM wireless network is essentially important into its success. The standard mobile internet protocol (mobile IP) offers a basic mobility management solution; however, a number of drawbacks limit its ineffectiveness in terms of scalability, locality, load balancing, route optimization, fault tolerance, etc. 


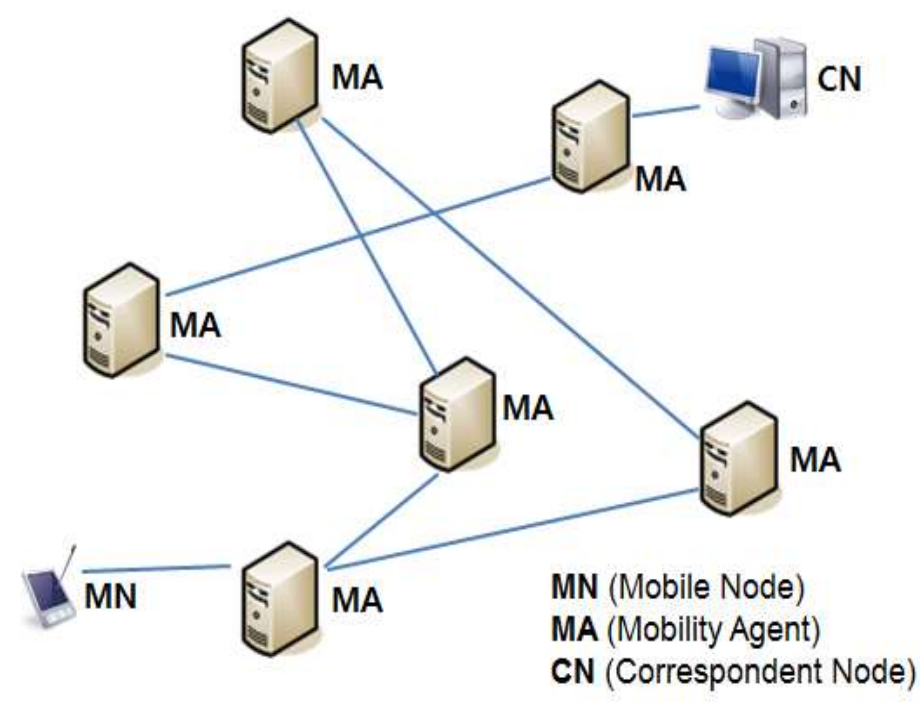

Figure 1. Peer-to Peer Mobility Management

The peer-to-peer (P2P) mobility management (shown in Figure 1) has addressed some issues of disadvantages raised by the standard mobile IP. The sensor gateways can be organized into P2P networks to improve the scalability, availability, locality, self-administration, and performance of the standard mobility managements.

\subsection{Centralized Mobility Management}

The centralized mobility management solutions in supporting the handovers of sensors and mobile devices in the SHM wireless network utilizes a central mobility anchor which is responsible for the management and control of sensors and mobile devices as well as on the routing of structural health data [11]. The structural health data however will be required to traverse the central mobility anchor making the data traffic suboptimal and can lead into overloading of network resources to the central mobility anchor. The overview of the centralized mobility management is shown in Figure 2.

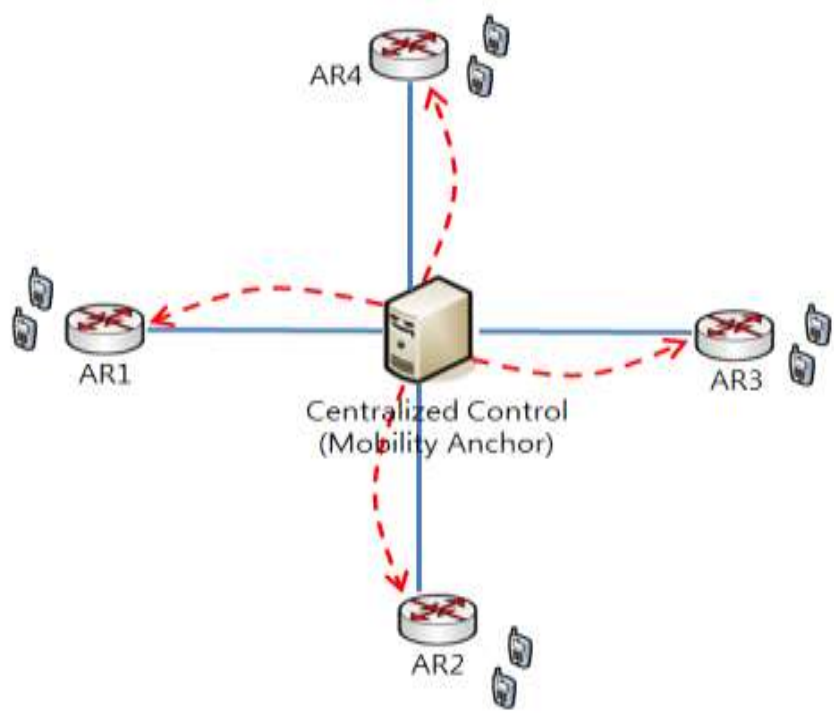

Figure 2. Centralized Mobility Management 
The approach can be considered as manageably efficient since all of network controls are anchored into a single point, but, some considerations can limit the efficiency of handovers and mobility of sensors and mobile devices in the wireless network. Such limitations include low scalability, reliability (central mobility anchors are susceptible to single point of failure), suboptimal routing (structural data traffic sent by sensors must traverse the centralized mobility anchors), higher packet loss rate (congestion is expected on the centralized mobility anchors (sensor gateways), signaling overhead (a number of mobility related signaling must be shared by the mobile devices and wireless network entities during handover procedures), complex network deployment (it becomes more complex as all controls of structural health data traffic and mobility management will be managed by a single entity, security (the central mobility anchors can be the single point for attacks.

\subsection{Distributed Mobility Management}

The distributed mobility management (DMM) is an emerging mobility management paradigm that employs mobility anchors at the edge of the access networks closer to the mobile users. It is being worked out by the IETF working group aiming to address the limitations and drawbacks of the centralized mobility management support for handover operations $[12,13]$. It employs a distributed data plane where the distribution and routing of data traffic can become optimal since the mobility anchors are located very close to the mobile user terminals. The control and data infrastructures were managed by the mobility anchors and the $\mathrm{MN}$ is allowed to have multiple anchors.

The DMM deployment is classified into two frameworks, namely, the fully distributed model and the partially distributed model. Both the data and control planes are managed by the mobility anchors in the fully distributed model. Each access router (AR) that serves as the mobility anchor implements both the functions of LMA and MAG of the networkbased mobility protocol. The mobility anchor serves as an LMA that anchors and routes multimedia traffic to the $\mathrm{MN}$ and serves as a MAG that receives the tunneled multimedia traffic from the MN.

On the other hand, the data and control planes are separated in the partially distributed DMM model, and only the data plane is distributed by the mobility anchors while the control plane is kept centralized. This setup was adapted in software defined networks. The control plane is managed by a particular mobility management entity (MME) (e.g., as in $3 \mathrm{GPP}$ networks), while the data plane is distributed by the mobility anchors.

\section{Software Defined Networking Overview}

The emergence of Software Defined Networking (SDN) has become a significant contribution in mobility management support for wireless network systems. The paradigm separates the control and data infrastructures aiming to optimize the transmission of critical structural information and manage the mobility support for sensors and mobile devices [14]. It is founded by the Open Networking Foundation (ONF) which aims to transform the disruptive wireless network systems to become directly programmable, agile, centrally managed, programmatically configured, and open standards-based or vendor-neutral $[14,15]$. It has also enabled the network administrators to programmatically initialize, control, transform, and manage the network system behavior dynamically through open interfaces and provide functional abstraction of the underlying infrastructure.

The SDN architecture is considered to be directly programmable (data forwarding was separated from network control); agile (the transmission of data is dynamically adjusted through abstraction of forwarding control); centrally managed (network resources can be maintained by software-based SDN controllers); programmatically 
configured (configuration and management of network system resources are allowed through dynamic and automated SDN programs); open standards-based and vendorneutral (network design and operation were simplified) [16].

Figure 3 shows the overview of the SDN architecture wherein it is generally divided into three planes, the data, control, and application planes.

- Application plane. The SDN applications (e.g., traffic management system) uses application programming interfaces (APIs) or interface drivers in communicating with the Control plane.

- Control plane. The SDN controller provides services to the SDN applications and controls the operations of the network elements in the Data plane. It dictates the direction of the flow of structural information traffic to optimize its delivery to the intended users.

- Data plane. The data plane is comprised of network elements and is controlled by the SDN controller through Control-Data-Plane Interface (CDPI) agent.

The overall management for assigning each network element with its own SDN controller is provided by the Management and Admin plane as well as setting up the configuration policies between SDN controller and applications such as the proposed information transmission system and bridge traffic monitoring systems [15].

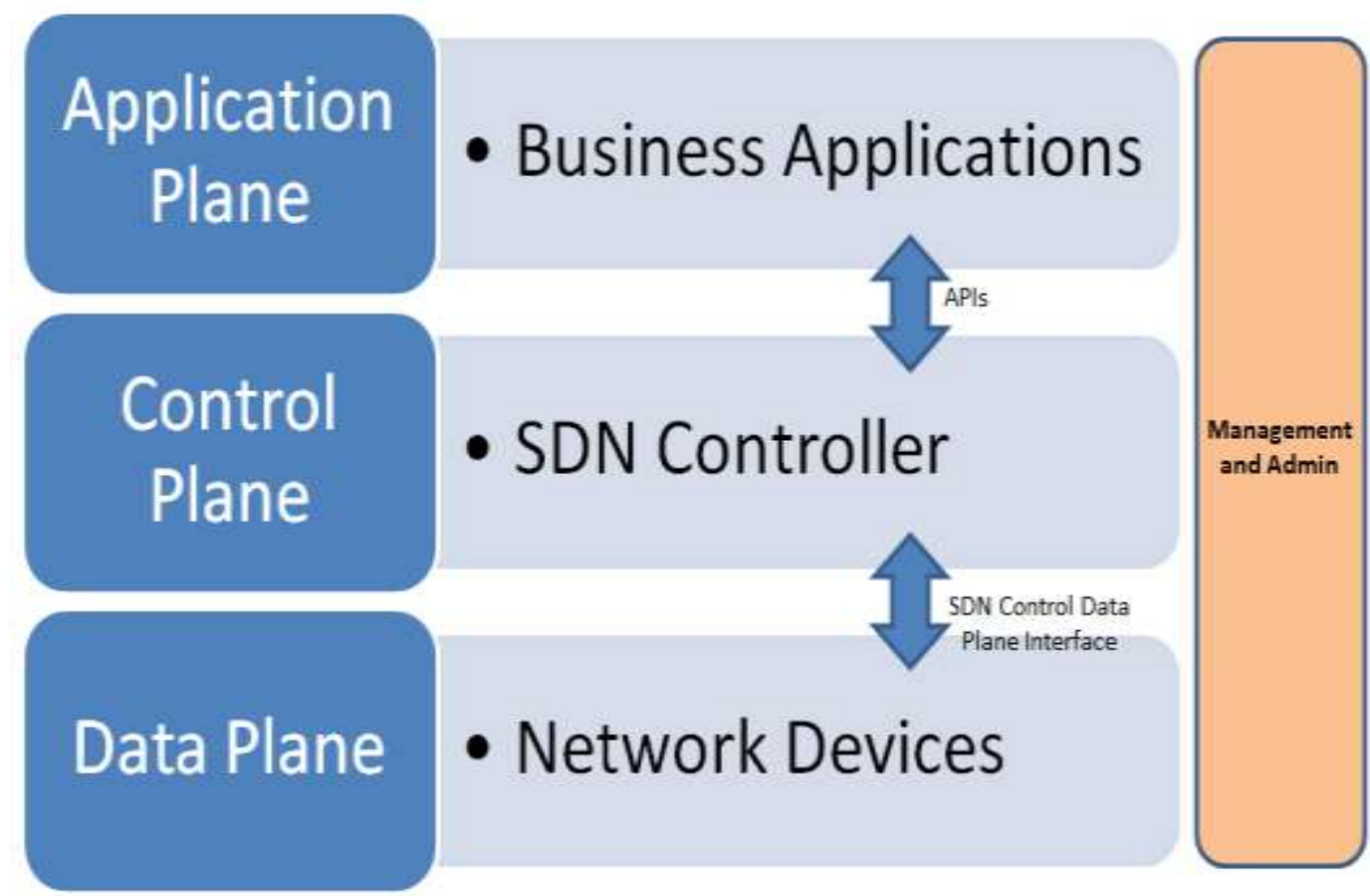

Figure 3. Overview of Software-Defined Networking Architecture

In the proposed wireless sensor information transmission system, the SDN implementation separates the network system that directs the flow of critical structural information traffic (i.e., where to send the IP packets) which is referred to as the SDN controller or control plane and the lower layer underlying systems that is responsible for forwarding the structural information traffic to the designated mobile terminal (i.e., vehicles passing the bridge) which is referred to as the data plane. The centralized control can still be implemented in such a way that an SDN 
controller is responsible on the overall control of the network resources. It is also responsible for providing information to the application plane through application program interfaces (APIs). In addition, it also manages all network devices on the data plane through a virtualized single logical switch that acts as the central controller via an SDN Control Data Plane Interface (CDPI). Thus, the transmission of critical information traffic will have a distributed mobility management support.

\section{DMM based Wireless Sensor Information Transmission}

This paper deals with the analysis of the integration of SDN as distributed mobility management support for wireless sensor transmission systems. It is designed to address the social issues of the safety and efficiency of SOC public structures specifically on bridges. The system is comprised of the geographical installation of distributed smart sensors in order to capture structural data of bridges, occurrences untoward incidents, as well as collecting information for unusual weather events such as early morning fog, earthquakes, and other natural calamities that can trigger any bridge failures. The large amount of collected and measure data is then filtered by the smart sensing device before it transmits into the distributed routers so that only critical information can be extracted and analyzed. The collected signals by the smart sensors will be analyzed, in such a way that optimal or critical data signals will be separated from insignificant data signals using a genetic signal filtering bank as depicted in Figure 4. In this way, only critical signals (important structural health data, triggering events such as vehicular accidents, scour, and earthquakes) will be transmitted by the smart sensors to the receiving routers and disregarding other insignificant signals. This can result into minimized data traffic across the wireless network, thus, improves its efficiency.

Whenever the routers have received the critical data sent by the smart sensors, the receiving router determines the most optimal route in delivering the received signals by checking the bandwidth of every transmission path. This can ensure an efficient delivery of critical information going to the home agent for analysis which will be used for an effective structural health monitoring.

Moreover, the vehicles passing the bridge will be acting as mobile nodes in a wireless network that are capable of receiving real-time traffic condition as continuously monitored by the proposed transmission system. A key can be used by the vehicle's smart device in registering into the nearest possible router as they pass by the bridge wherein its mobility management follows the concept of software defined networks. This enables a distributed mobility management support based on SDN. The vehicle's smart device will be equipped with a seamless connectivity to enable its drivers to access into real-time traffic conditions delivered by the proposed system. The vehicle's smart device will be capable of moving its registration from router to router as it goes through the bridge, thus, any occurrence of naturally caused calamities or unusual events such as vehicular accidents or congestion of cars can be updated to the car drivers. 


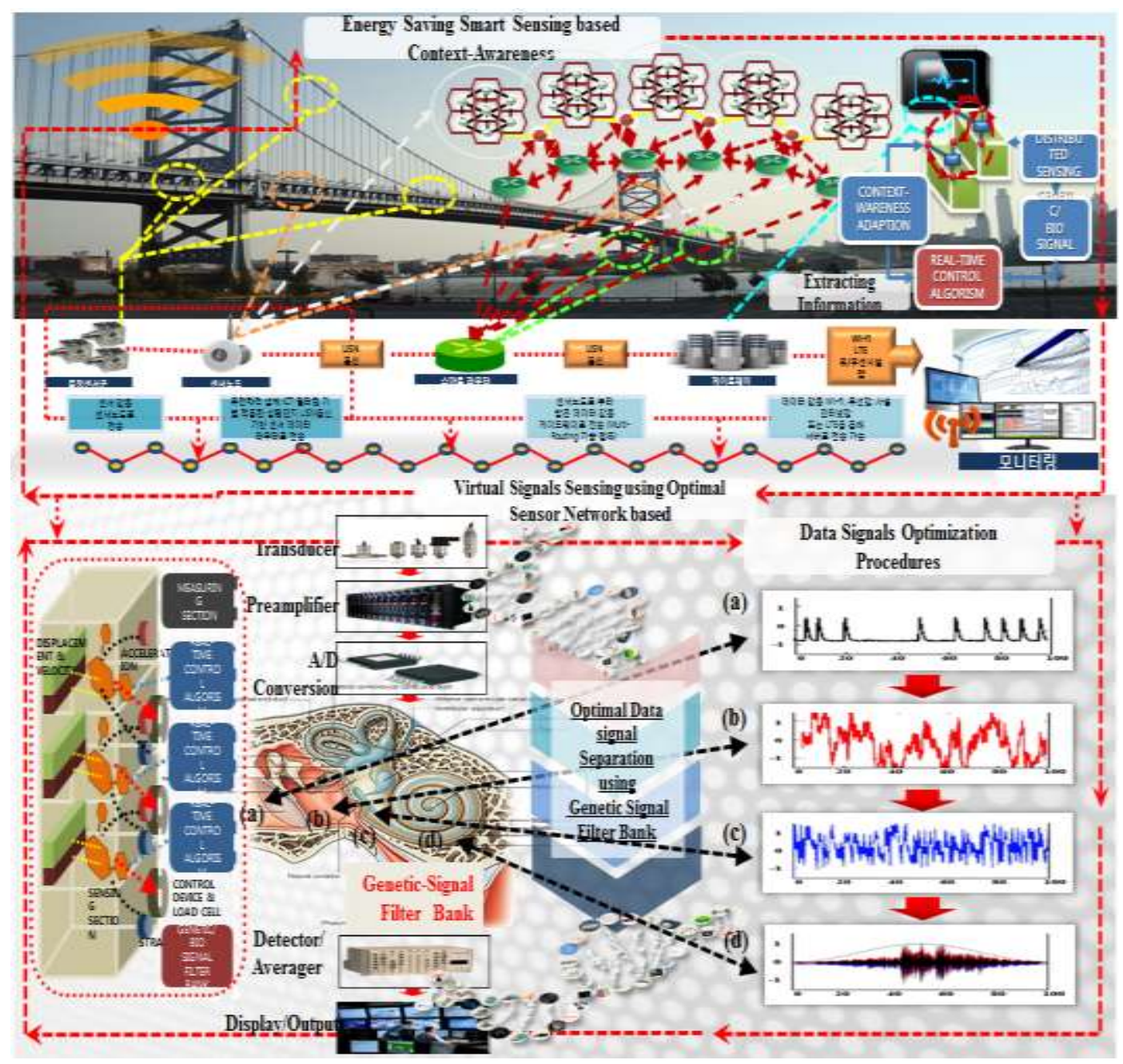

\section{Figure 4. Context-Aware based Energy saving Genomics-Bio Transmission System}

The separation of data infrastructures (delivery of critical signals) from its control infrastructures has allowed a more efficient management, provisioning and configuration of wireless system. The network system and the flow of critical structural health signals can be managed in a centralized manner resulting to a seamless mobility support for vehicles. The SDN based DMM support for the delivery of critical structural health data across the wireless sensor information system is shown in Figure 5. It employs APIs in order to implement network services that include routing, multicasting, security policies, network resources access control, bandwidth management, directing traffic flow, QoS, processor and storage optimization, energy usage, and all forms of policy managements. 


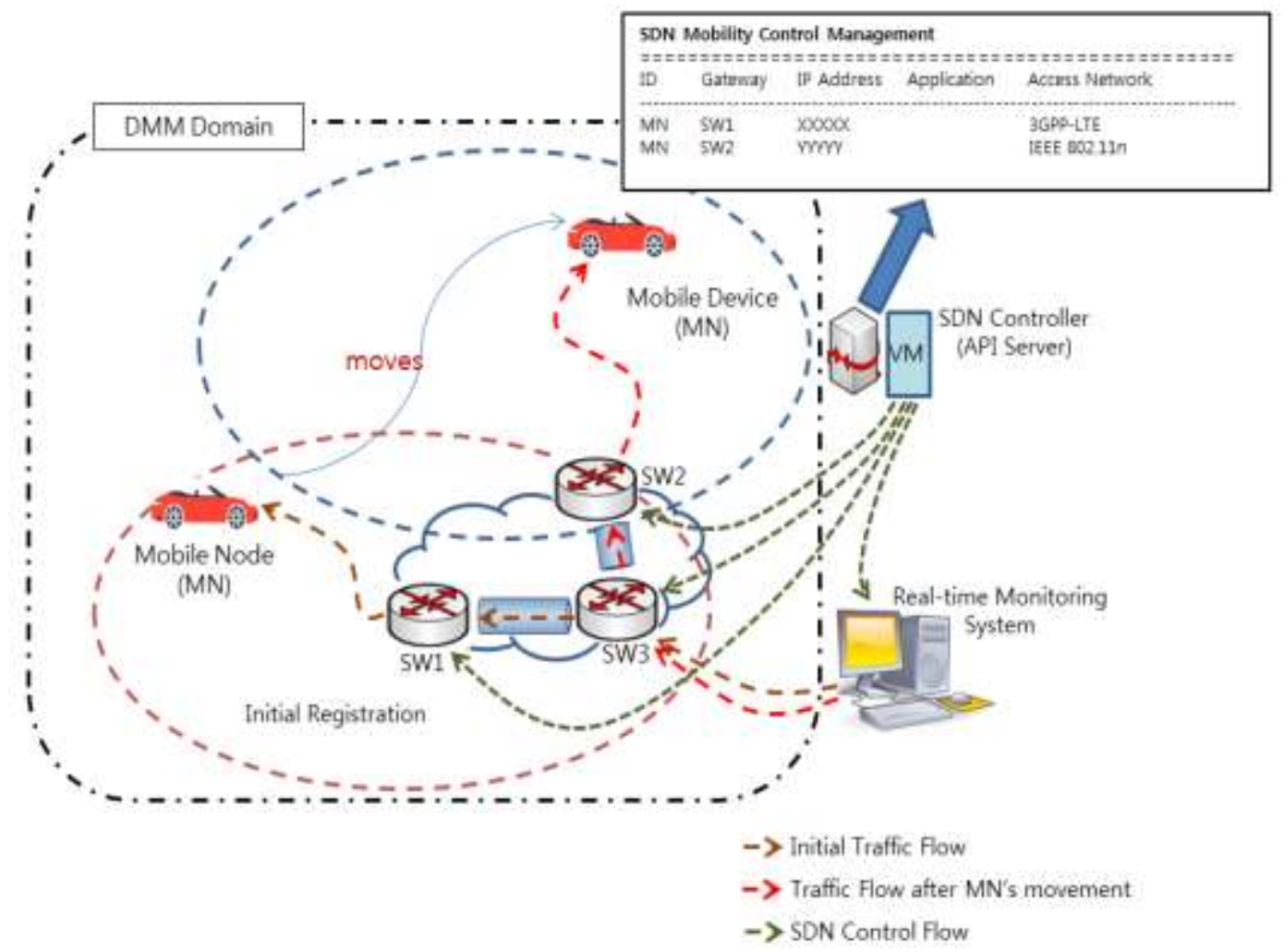

Figure 5. SDN based Distributed Mobility Management Support for Wireless Sensor Information Transmission System

The SDN controller is responsible for the management and decision making of the destination of critical structural health data among the network elements which are only capable of data forwarding functions, thus, optimizes its routability .

The filtering algorithm, the distributed routing, and the programmable control for the flow of critical information of structural data will be an enormous lift on delivering a robust structural health monitoring and real-time traffic and unusual weather updates specifically for bridges. The proposed wireless sensor information transmission system can guarantee the safety to bridge commuters as well as in the improvement of the maintenance of other SOC public structures.

\section{Conclusion}

This paper has presented an analysis of an SDN based distributed mobility management support for wireless sensor information transmission system designed for the monitoring of SOC public infrastructures specifically on bridges. This scheme implements the separation of the data and control infrastructures in order to optimize the delivery and routing of critical structural information. A filtering algorithm to separate the critical structural information from insignificant sensor signals have reduced the congestion of data traffic across the wireless network and has allowed a significant optimization for routing critical structural information.

\section{Acknowledgments}

This research was supported by Basic Science Research Program through the National Research Foundation of Korea (NRF) funded by the Ministry of Education (2018R1D1A1B07044131). 


\section{References}

[1] J. P. Lynch, "An overview of wireless structural health monitoring for civil structures", Philosophical Transactions of the Royal Society, vol. 365, (2007), pp. 345-372, doi:10.1098/rsta.2006.1932, Published online: 14 December 2006.

[2] Y. Wang, J. P. Lynch and K. H. Law, "Wireless Sensing, Actuation and Control - With Applications to Civil Structures", In: Smith I.F.C. (eds) Intelligent Computing in Engineering and Architecture, EG-ICE 2006, Lecture Notes in Computer Science, vol. 4200, (2006), Springer, Berlin, Heidelberg, https://doi.org/10.1007/11888598_60.

[3] P. Bertin, S. Bonjour and J. Bonnin, "Distributed of centralized mobility", In Proceedings of IEEE Global Telecommunications Conference (GLOBECOM 2009), IEEE, (2009), pp. 1-6.

[4] H. A. Chan, H. Yokota, J. Xie, P. Seite and D. Liu, "Distributed and Dynamic mobility Management in Mobile Internet: Current Approaches and Issues", Journal of Communications, Academy Publisher, vol. 6, no. 1, (2011), pp. 4-15.

[5] S. J. Cho, C. -B. Yun, J. P. Lynch, A. T. Zimmerman, B. F. Spencer Jr. and T. Nagayama, "Smart Wireless Sensor Technology for Structural Health Monitoring of Civil Structures", Steel Structures, vol. 8, (2008), pp. 267-275.

[6] C. J. Bernardos, M. Gramaglia, L. M. Contreras, M. Calderon and I. Soto, "Network-based Localized IP mobility Management: Proxy Mobile IPv6 and Current Trends in Standardization", Journal of Wireless Mobile Networks, Ubiquitous Computing, and Dependable Applications (JoWUA)(Special issue: Advances in Wireless Mobile and Sensor Technologies), vol. 1, no. 2/3, (2010), pp. 16-35.

[7] https://en.wikipedia.org/wiki/Tacoma_Narrows_Bridge_(1940), Retrieved: (2018) May 21.

[8] NTSB, "Collapse of I-35W Highway Bridge, Minneapolis, Minnesota, August 1, 2007", National Transportation Safety Board, https://www.ntsb.gov/investigations/AccidentReports/Reports/ HAR0803.pdf, Retrieved: (2018) May 21.

[9] https://www.timesunion.com/local/article/30-years-ago-Bridge-collapse-kills-10-11045976.php, Retrieved: (2018) May 21.

[10] Reuters, "32 Reported Dead as Bridge Collapses in Seoul", 1994, October 22, https://www.nytimes.com/1994/10/22/world/32-reported-dead-as-bridge-collapses-in-seoul.html, Retrieved: (2018) May 21.

[11] https://2wtech.com/centralized-mobility-management/, Retrieved: (2018) May 21.

[12] J. C. Zúñiga, "Distributed Mobility Management: A Standards Landscape", IEEE Communications Magazine, vol. 51, no. 3, (2013) March, pp. 80*7.

[13] B. Sarikaya, "Distributed Mobile IPv6", IETF Internet-Draft, (2012) February.

[14] ONF White Paper, "Software-Defined Networking: The New Norm for Networks", (2012) April 13, https://www.opennetworking.org/images/stories/downloads/sdn-resources/white-papers/wp-sdnnewnorm.pdf.

[15] ONF White Paper, "SDN Architecture Overview", (2013) December http://www.opennetworking.org/wp-content/uploads/2013/02/SDN-architecture-overview-1.0.pdf.

[16] "Software-Defined Networking (SDN) Definition", https://www.opennetworking.org/sdn-definition/, Retrieved: (2014) October 26. 